\title{
Errors in non-contact sensor measurements due to misalignment and scanning methodology
}

\author{
Zachary Warlick ${ }^{1}$ and Reuven Katz $^{2, \star}$ \\ 1 Department of Mechanical Engineering, University of Michigan, Ann Arbor, MI, USA \\ 2 Mechanical Engineering Department, Technion, Israel Institute of Technology, Haifa, Israel
}

Received: 25 May 2015 / Accepted: 16 July 2015

\begin{abstract}
Non-contact sensors are becoming an increasingly popular tool for use in dimensional measurement, inspection and quality control. However, there exist a number of challenges associated with the use of these non-contact sensors in dimensional inspection systems. One important challenge is to understand the associated measurement errors and determine the objective accuracy of such an inspection system. This paper examines the effect of misalignment and scanning methodology on the measurement accuracy of a non-contact dimensional inspection system. It is important to understand this topic since the majority of optical sensor manufacturers do not supply information about the alignment between the optical line of sight and the mechanical interface of the sensor or measurement system. We prove the evidence of this measurement error through both experimental results and simulation. Finally, by using a constant standoff measurement method we succeeded to show that the majority of inherent misalignment error can be eliminated.
\end{abstract}

Keywords: Metrology, non-contact inspection, error analysis, scanning methodology

\section{Introduction}

Non-contact sensors are becoming widely used in dimensional metrology, inspection and quality control. Measurement systems employing non-contact sensors offer a variety of benefits over traditional, contact-based measurement systems such as coordinate measurement machines (CMMs). Non-contact systems can gather large quantities of data in small times and perform measurement without physical contact. By eliminating physical contact, non-contact sensors allow for the measurement of highly sensitive parts that could be potentially damaged or altered by a contact stylus. Additionally, many non-contact sensors are capable of measurement accuracy on the scale $5 \mu \mathrm{m}$ or better [1]. There exist a variety of different non-contact measurement technologies that are described in several review papers [1-3]. These extensive papers review most of the available non-contact sensors and measuring techniques. In general, non-contact sensors fall into one of three main categories: area sensor, line sensor, or point sensor.

A wide variety of non-contact point sensors have been developed and many have become commercial products. These commercial products are typically integrated into existing measurement systems, for example, the triangulation sensor described in reference [4] that was integrated into a coordinate measurement machine (CMM).

^ Correspondence: reuvenk@technion.ac.il
The performance of these sensors is generally examined as a part of a commercialized measurement system, and no independent, objective standard has been presented to characterize the performance of the sensors or performance of the overall non-contact measurement system. These non-contact point sensors create system design challenges and require extensive calibration work.

Limited work has been published to characterize objectively non-contact sensor performance due to the wide range of technologies used in the sensors and the range of intended applications of the sensors $[2,3]$. Similarly, little work has been published to characterize objectively the performance of dimensional inspection systems using non-contact sensors. An interesting example of a discussion and analysis of error budget in measurement system is presented in reference [5]. A laser ball bar, consisting of a laser interferometer aligned with a telescoping ball bar, was used for mapping the volumetric positioning errors of machine tools. The authors analyze interferometry errors, sphere-line misalignments, and optical rotation errors to show the combined effect on measurements. The error analysis of the experimental system that was used in this study is presented in reference [6].

There are a number of challenges associated with the accurate characterization of non-contact sensor performance in dimensional inspection systems. In order to ensure the overall accuracy of any non-contact dimensional inspection system, it is necessary to understand the combined effects of a variety of error sources. Errors can arise 
from any of the associated sub-systems including the sensor, motion stages, controls and software. Furthermore, there is potential for error in the setup and operation of a dimensional measurement system, such as calibration error, alignment error and operator error.

In the design and analysis of any dimensional inspection system all of these potential error sources must be carefully considered, however this is especially true when using a non-contact sensor. For example, when considering alignment error, the alignment of the sensor with respect to the machine axes is one of many important contributing factors to system accuracy. Also, the operation of the system, specifically the scanning methodology, can have a significant impact on measurement system performance [7]. Research findings have been published in the field of scan plan generation using range-finding non-contact laser sensors [8-10]. The algorithm applied by registration software will have an impact on the result that is presented by the software. A full understanding of the underlying algorithm is needed to ensure that the actual registration process matches the expected registration process.

The combined effects of these conditions are not fully considered in many situations where non-contact sensors are utilized. If not considered, dimensional measurement systems may not yield the intended measurement results. The result of scanning under such conditions is measurement data with significant systematic error. These systematic effects may lead to an invalid assessment of the quality of the part being inspected. This substantial error is unacceptable when the expected magnitude of system accuracy is on the order of the sensor error $(5 \mu \mathrm{m}$ or better). Several studies discuss these errors in non-contact laser scanning.

A thorough analysis of digitizing errors has been presented [11] and methods for error correction based on experimental process have also been discussed [12]. A methodology for comparison of laser digitizing relative to contact measurements was performed for validating the accuracy when measuring dimensional and geometrical tolerances [13]. A "Virtual ball" method [14] was developed for the interpretation of laser measurements of mechanical parts, as if they were performed by a standard coordinates measurement machine (CMM).

Our study addressed development of technologies required for a prototype high-accuracy, high-density, noncontact dimensional inspection system [15]. This reference summarizes an extensive experimental benchmark testing of three high-accuracy range-finding point-sensors and includes discussions of: angular measurement, measurement precision throughout working range, sensor precision and repeatability, step height measurement, and oversampling.

This paper examines the effect of misalignment and scanning methodology on the measurement accuracy of a non-contact dimensional inspection system. The existence of this measurement error is demonstrated through both experimentation and simulation. A method for eliminating this inherent measurement error is suggested and the improvement in accuracy is examined. Section 2 presents the problem statement and Section 3 provides a brief description of the experimental set-up. Section 4 shows the experimental results and Section 5 discusses the conclusions.

\section{Problem statement}

The research presented in this paper was initiated after measurement errors were observed. During experimentation a non-contact laser point sensor and a 3-axis motion stage setup were used to measure a gauge ball. By measuring a known "perfect" artifact, the goal was to examine the accuracy of the measurement system. However, when the measurement data was reviewed, a significant and distinct error pattern was observed. Since the source of the phenomenon was unknown, research began by first examining the individual measurement system components. These components were the sensor, registration software, scanning methodology and sensor alignment. After understanding these individual items, we explored the combined effect of all of their associated error sources within a non-contact dimensional inspection system.

\subsection{Point sensor}

This paper is concerned primarily with the errors associated with point sensor measurement. Point sensors measure the distance between the sensor and a single point on the part surface. Specifically, the point sensors considered while performing this research had standoff (the distance from the sensor to the middle of the working range) distances on the order of $15 \mathrm{~mm}$ to $30 \mathrm{~mm}$ and working ranges (the range of distances within which the sensor can take measurements; also referred to as the "depth of view" or "focal depth") on the order of $1.5 \mathrm{~mm}$ to $3 \mathrm{~mm}$. The accuracy of such point sensors as reported by OEM specifications is on the order of $0.001 \mathrm{~mm}$. While this paper is directly related to the use of point sensors, the results of this paper may also have applications to the use of line and area sensors.

\subsection{Registration software}

After measurement data is acquired it must be analyzed to gain some useful information. When performing dimensional inspection of parts, the data is generally analyzed through a comparison with a CAD model. The data from a non-contact measurement system is generally obtained in the form of a cloud of points. This cloud of points is then matched onto the CAD model using a process known as registration [16]. Deviations of the measurement data from the ideal model can then be seen.

The registration software examined in this work used a best-fit algorithm to match the cloud of points to the CAD model. There are additional methods of registration which involve the use of a datum or registration features. Use of these registration methods may cause slightly different measurement results than the best-fit registration method. 


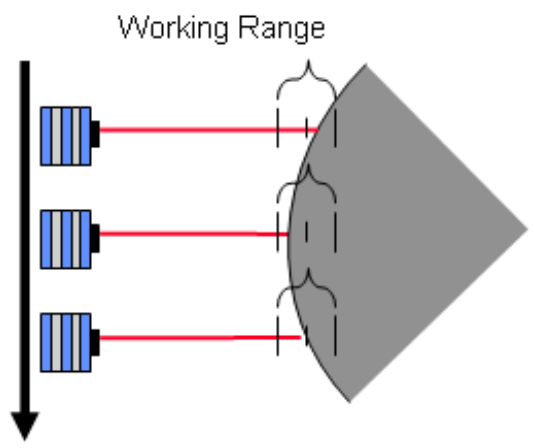

Fig. 1. Traditional scanning method; non-constant distance between the part and the sensor.

However, similar conclusions regarding measurement errors of the system can be drawn using any registration method.

\subsection{Scanning methodology}

There are various scanning methodologies used to perform dimensional measurement of a complex geometry using a non-contact sensor. Scanning can be performed while maintaining a constant distance (ideally the standoff length) between the sensor and the part surface [10]. This method of scanning is similar to the method used by CMMs. In the case of a CMM, the constant standoff distance is the length of the probe stylus.

Additionally, scanning can be performed while not maintaining a constant distance. During this traditional scanning method, the non-contact sensor is moved in a predetermined path. The part geometry varies within the working range of the sensor as seen in Figure 1 and thus the distance between the sensor and the surface of the part varies.

Work has been performed in the area of scan plan generation using non-contact sensors. The suggested scan plans utilize the traditional scanning method in which there is a non-constant standoff. The plans only ensure that the part surface remains within the working range of the sensor.

These traditional scanning methods often take into account the effect of measurement angle (the angle defined between the sensor beam the surface normal of a part) and ensure that the measurement surface remains in the working range, however they fail to analyze the effects of potential misalignment and measurement angle in conjunction with their proposed methods. Furthermore, the described methods of inspection planning primarily examine the use of line or area sensors, whereas this work is concerned with the use of more accurate point sensors.

\subsection{Sensor alignment}

Alignment of the non-contact sensor beam with respect to the machine axes of the dimensional measurement system should be considered. If the orientation of the beam
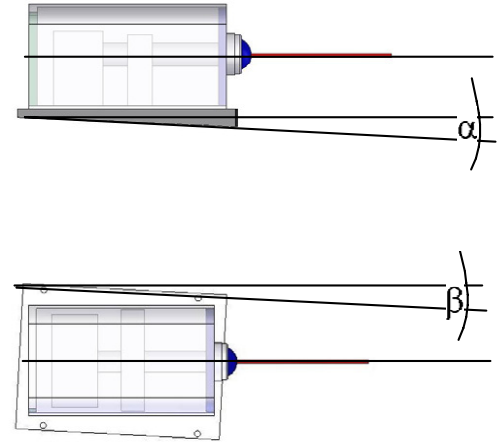

Fig. 2. Misalignment errors, $\alpha$ (side view) and $\beta$ (top view), between sensor body and machine axes.

is unknown, then the intended measurement may not be the same as the acquired measurement. In many instances, the alignment is never verified, yet the alignment of the sensor is assumed to be correct. There are various possible sources of beam misalignment within the measurement system. First, the beam may be misaligned within the sensor housing if the optics are not manufactured and assembled accurately with respect to the housing datum surface. Second, there may be some misalignment between the sensor housing and the machine axes due to the mechanical coupling of the bolts (Fig. 2). Finally, there may be some misalignment between the machine axes.

Research on the topic of probe positional alignment with respect to machine axes has been examined [17]. The methods of alignment are based in sound theory and have been verified through simulation. However, the inherent accuracy of current non-contact sensor technology is insufficient for the practical application of the methods. Specifically, the non-contact sensors were not able to perform measurements with the necessary degree of accuracy at large measurement angles to use the proposed alignment methods.

Sensor misalignment alone may not contribute large errors to a non-contact measurement system. However, misalignment in conjunction with the scanning methodology must be considered. It is shown in this paper that misalignment coupled with traditional scanning methodologies can cause significant measurement errors when scanning at large measurement angles.

In summary non-contact sensor performance has not been objectively defined and is provided only through proprietary OEM specifications. Furthermore, the error characterization of a dimensional inspection system using such a non-contact sensor remains unstudied. Through examining the above mentioned system components of a noncontact dimensional inspection system we characterize a unique source of measurement error.

\section{Experimental set-up}

\subsection{Non-contact sensor testbed}

The non-contact sensor testbed is a coordinate measuring machine (CMM) that was customized and built 


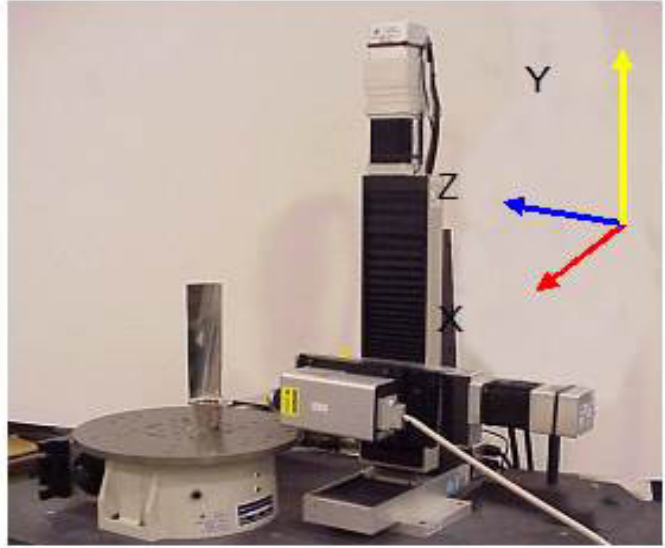

Fig. 3. Non-contact sensor testbed.

in-house for the purpose of inspecting the complex geometries present in turbine blades and like parts (Fig. 3). The Testbed employs a non-contact sensor mounted on Aerotech linear stages, providing 3D movement. A rotary table enables 360-degree rotation of an inspected part, generating a fourth degree of freedom to the Testbed. The stages were assembled by the manufacturer and then mounted and aligned on a granite table. The stages and probe controllers are connected to and monitored by a PC.

The non-contact sensor testbed was used as a base for many of the sensor performance tests. Much like a CMM, the Testbed allows the user to measure and record the $X$, $Y$ and $Z$ coordinates of points on the surface of a workpiece. The Testbed was used in this fashion to perform line scans and raster scans (area scans) of research artifacts. Because the Testbed employs a non-contact sensor, it is not limited to discrete, point-by-point scans as with a traditional CMM. Scans taken with the Testbed can be done continuously, limited only by the speed of the motion stages, sampling rate of the sensor and the desired density of data.

Testing has been done using a Renishaw interferometer to determine the magnitude of error introduced by the motion stages of the Testbed. A straightness test was performed on the Testbed $x$-axis. The straightness test was performed because errors of the $x$-axis motion stage (sideto-side "wobble") affect the sensor measurements in the $z$-direction. Thus, straightness errors of the $x$-axis will be the most significant of the motion stage error components when performing a simple line scan.

The Testbed was moved along the entire length of travel, while the interferometer measured movement in the $Z$-direction. Multiple passes were made and the results showed a repeatable bowing along the length of travel. This systematic error is believed to be mechanical in nature and has amplitude of approximately $0.011 \mathrm{~mm}$ when moving along the full $250 \mathrm{~mm}$ length of the motion stage. The measurement noise is approximately $0.003 \mathrm{~mm}$ in amplitude. These known measurement errors were considered when analyzing all experimental results.

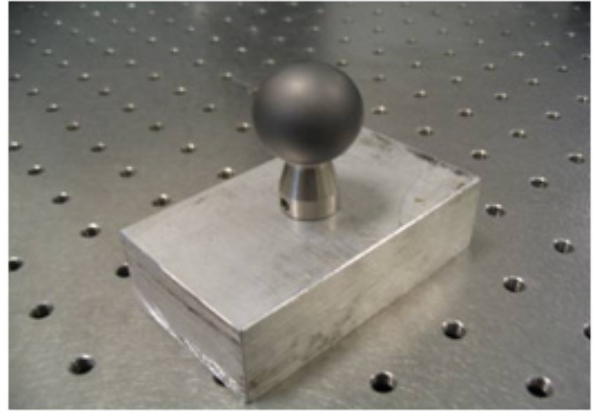

Fig. 4. Renishaw matte ball.

\subsection{Optimet smart conoProbe}

The Optimet Smart ConoProbe was one of the three noncontact sensors selected for the extensive benchmark testing performed at the University of Michigan. The other two sensors were the Stil Optical Pen and the Keyence LK-G10. The experiment presented in this paper used the Smart ConoProbe with a $25 \mathrm{~mm}$ lens. Before using the sensor, it was allowed to warm up for 30-40 min to allow for thermal expansion. During the different experiments, the Smart ConoProbe settings were adjusted to achieve the best results. The artifact that was tested was the Renishaw Matte Ball shown in Figure 4.

\section{Results}

\subsection{Error summary and analysis}

The effect of sensor misalignment and scanning methodologies was examined through the measurement of a gauge ball. Scanning was performed using the traditional, nonconstant distance scanning methodology, in which a constant distance between the sensor and part was not maintained. The surface of the gauge ball was scanned by moving the sensor in a raster scan motion.

The cloud of points acquired through the scan was then registered to a $\mathrm{CAD}$ model of the gauge ball. The deviation of the cloud of points from the known geometry of the gauge ball was examined. The resulting errors of the measurement data from the model can be seen in Figure 5.

A distinct error pattern can be seen in the cloud of points. Regardless of the scanning conditions, a common trend was observed in all scans. After registration, this pattern was observed in the deviation of the measurement points from the solid model. There always appeared a patch of measured points above the CAD model (darker blue points) and a patch of measured points that remained below the CAD model (lighter yellow points).

The cause of the error pattern in the gauge ball measurements can be explained as the collection of a number of factors from both the measurement system and the data analysis (registration). Errors associated with the measurement system (from sources such as the scanning methodology, misalignment of the measurement system, and measurement angle) as well as the effects of data 


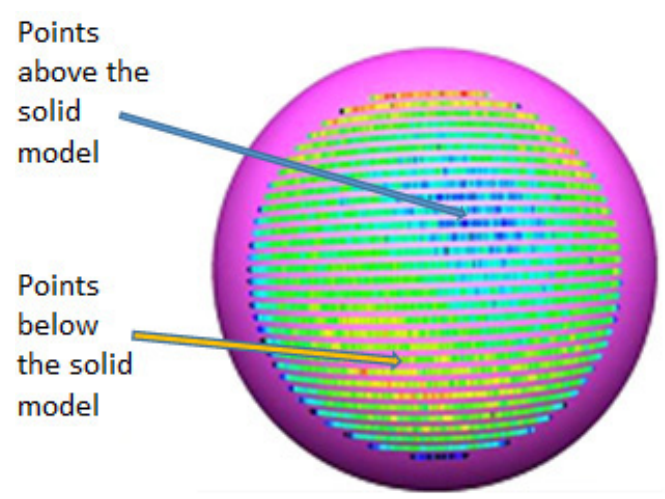

Fig. 5. Deviation of a measured cloud of points from CAD model.

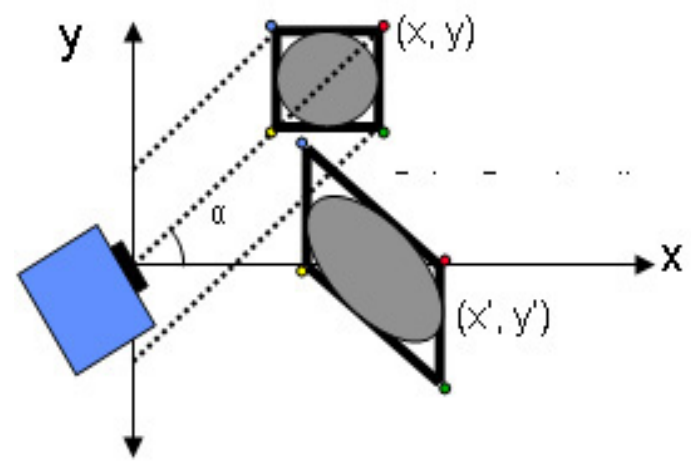

Fig. 6. Example of transformation caused by misalignment in a measurement system.

analysis (registration of the erroneous cloud of points) are contributing factors. We will first examine the errors in measurement that occur during data collection.

\subsubsection{Measurement system errors}

Using the traditional non-constant distance measurement method, errors arise when there is misalignment of the measurement system. We show the effect of using traditional scanning methods with a misaligned system through a base example. For the purpose of this example we assume that we are measuring a perfect artifact and that our sensor is perfect.

The 2D example of the resulting error can be seen in Figure 6. Under perfect operating conditions, the motion stage direction ( $y$-axis) and the sensors optical path $(x$-axis) are perpendicular. In this situation, the accurate $(x, y)$ coordinates of the box are measured. Examine, for example, the upper-right corner of the box before transformation. The $x$-coordinate of the corner is the distance of the point along the $x$-axis (measured by the sensor), and the $y$-coordinate of the box is the distance of the point along the $y$-axis (measured by the motion stages).

However, if the optical path is misaligned from perpendicular by some angle, $\alpha$, then the resulting measurements

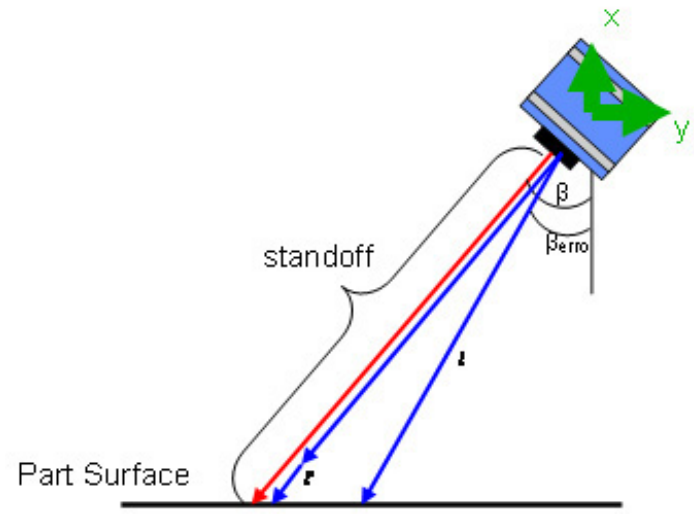

Fig. 7. Measurement errors as affected by misalignment and measurement angle using traditional scanning methods.

undergo a transformation and appear skewed. In this situation, the incorrect $\left(x^{\prime}, y^{\prime}\right)$ coordinates of the box are measured. The relationship between $(x, y)$ coordinates and $\left(x^{\prime}, y^{\prime}\right)$ coordinates is given by equation (1), and can be seen in Figure 6.

$$
\left[\begin{array}{l}
x \\
y
\end{array}\right]=\left[\begin{array}{ll}
\cos (\alpha) & 0 \\
-\sin (\alpha) & 1
\end{array}\right]\left[\begin{array}{l}
x^{\prime} \\
y^{\prime}
\end{array}\right] .
$$

Examine again the upper-right corner of the box before transformation. To measure that same point with sensor misalignment, the sensor records the $x^{\prime}$ position which is the diagonal distance from the sensor to the upper-right corner. The $y^{\prime}$-position is recorded by the motion stages.

Thus, even measuring an ideal artifact, using a perfect sensor, there is inherent error due to misalignment in the system, which can cause errors. These errors become much more complex and larger as we include the effects due to measurement angle.

The measurement error seen when using the traditional, non-constant distance measurement method is further compounded by the fact that the sensor does not generally measure normal to the surface of the workpiece. When measuring the profile of a gauge ball, the measurement angle made between the sensor and the normal vector of the gauge ball varied within $\sim+40^{\circ}$. At large measurement angles the error due to misalignment is greatly amplified.

To explore the effect of misalignment at large measurement angles we look at the setup seen in Figure 7. When the measurement angle $\beta$, is known, we can accurately measure any point on the surface. However, when there is some misalignment, $\beta$ will vary, resulting in $\beta_{\text {error }}$. In the previous example, we examined the situation in which $\beta=0^{\circ}$ and $\beta_{\text {error }}=\alpha$. In this example, however, we wish to see how large measurement angles (i.e. large values of $\beta$ ) affect the error. In real life measurements, such as the gauge ball measurements, we expect to have a wide range of measurement angles.

Following the setup in Figure 7 and equations (2)-(4) below, we see that misalignment at large measurement angles results in an incorrect measurement. The incorrect 


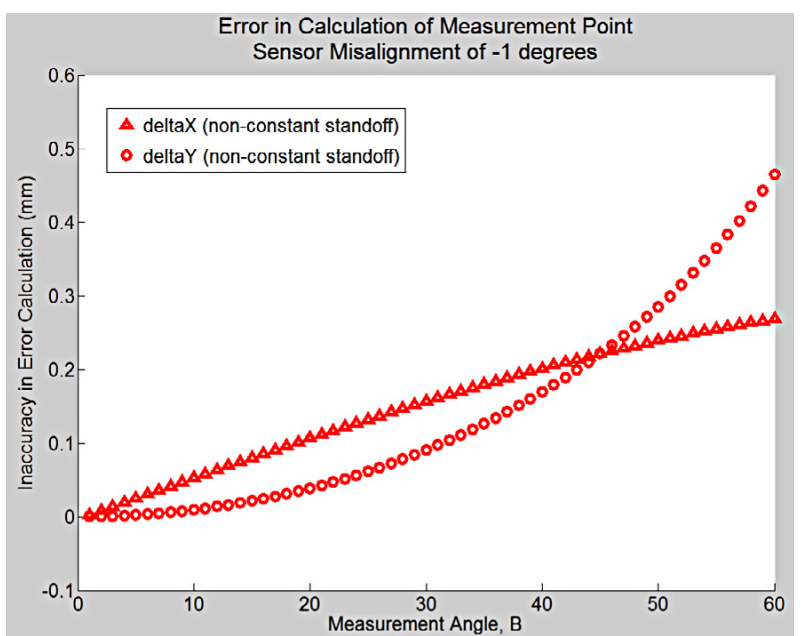

Fig. 8. Effect of measurement angle on measurement error, with a misalignment of $1^{\circ}$.

measurement is located at a distance $(\Delta x, \Delta y)$ from the true measurement location. As the measurement angle $\beta$, increases, the values of $\Delta x$ and $\Delta y$ also increase. The relationship between measurement angle and measurement location error, $\Delta x, \Delta y$, can be seen in Figure 8 below.

$$
\begin{aligned}
l & =\frac{\text { standoff } \times \cos (\beta)}{\cos \left(\beta_{\text {error }}\right)} \\
l^{\prime} & =\operatorname{standoff}-l \\
\Delta x & =l^{\prime} \sin (90-\beta) .
\end{aligned}
$$

It should also be noted that the geometry of the artifact surface may also affect the magnitude of the measurement errors. However this source of error is specific to each artifact. It is assumed for the purpose of this analysis that the curvature of the artifact is large and the value of $\beta_{\text {error }}$ is relatively small such that the surface can be reasonably approximated as linear.

\subsubsection{Registration}

After the measurement system errors were studied, research was performed to determine how registration of the erroneous cloud of points created the patterns shown in Figure 5. Previous analysis of the measurement system error (Sect. 4.1.1) showed that when measuring a circle with the misaligned system, the resulting measurement data would be an ellipse. Furthermore, when measuring a sphere, the resulting measurement data would be an ellipsoid.

We will consider the simple, $2 \mathrm{D}$ case of a line scan measurement of a perfect circle. The sensor is moving in a straight line, so only an arc of the circle is measured (Fig. 9a). This figure presents a top view of the scanner and a perfectly cylindrical rod. However, because the measurement system is skewed, the data captured by the sensor will not be a circular arc. The data captured by the sensor will be an elliptical arc (Fig. 9b). During the process of registration, a computer program tries to match

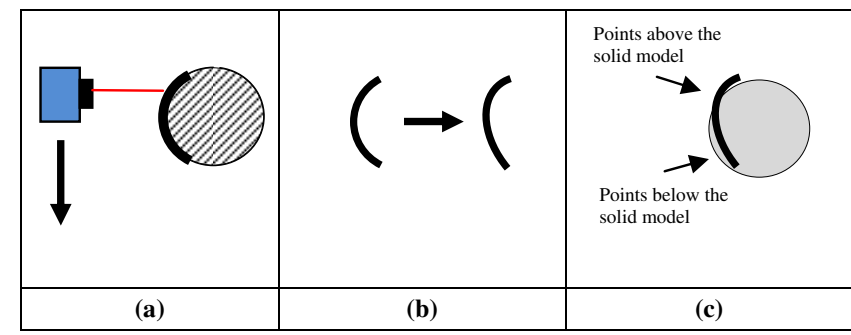

Fig. 9. Measurement and registration of skewed data.

the elliptical arc to the circular CAD model of the rod cross section (Fig. 9c).

By expanding this concept from a 2D line scan of a circle, to a 3D raster scan of a gauge ball, it can be seen how registration of the cloud of skewed points results in the patches seen in Figure 5. Recall that the registration software is based on a least squares algorithm. The least squares algorithm minimizes the sum of the squares of the distances from the points to the surface. Figure 9c shows two large patches in the middle of the scan data, one above the solid model surface (dark points in Fig. 5) and one below the solid model surface (bright points in Fig. 5). This finding is consistent with all experimental data and simulations.

It is important to note that the appearance of the patterns (Fig. 5) only applies to situations in which a free cloud of points is being registered to a solid model without the benefit of any known artifact datum or registration features. Without a datum or registration feature the registration of the cloud of points may be ambiguous, based only on the constraints of the best-fit algorithm. In the case where measurements are being performed with a datum this registration effect may be ignored.

\subsection{Simulation comparison}

By deliberately misaligning the sensor to a major degree $\left(\sim 15^{\circ}\right)$, a unique pattern similar to that found in experimental data (Fig. 5) was observed. However, the deviations seen in the deliberately misaligned scans were on the order of $+0.2 \mathrm{~mm}$ to $0.25 \mathrm{~mm}$. This is consistent with the assumption that misalignment was causing the error, as a minor misalignment $\left(\sim 1^{\circ}\right.$ or less $)$ caused an error of $+0.02 \mathrm{~mm}$ to $0.03 \mathrm{~mm}$; a large misalignment caused an error of $+0.2 \mathrm{~mm}$ to $0.25 \mathrm{~mm}$.

This error was simulated by generating a cloud of points as taken by a misaligned $\left(\sim 15^{\circ}\right)$ sensor and then registering the cloud of points to a solid model. Computer simulation of the misalignment showed nearly identical results to those witnessed in experimentation. The location of the error patches and the magnitude of measurement error were both consistent.

\subsection{Constant distance scanning method solution}

As can be concluded from the previous analysis and experimental results, the magnitude of errors associated with measurement using the traditional non-constant distance 
scanning method is prohibitive. Due to the experimental nature of the non-contact sensor testbed, misalignments of $\sim 1^{\circ}$ or less were not unexpected, however, in practice most CMMs and dimensional inspection machines are constructed and calibrated with a much higher degree of precision. Errors of this nature on a CMM would be orders of magnitude less than the errors described in this paper. However, if a professional measurement is not available, or, if the accuracy of a measurement system cannot be verified, a method of scanning is needed which would contribute measurement errors on the scale of a few microns or less.

A measurement method that maintains a constant distance between the sensor and the measurement part was examined. Using a non-contact sensor to perform measurements, while maintaining a constant distance between the sensor and the part, is analogous to performing measurement using a traditional CMM. For each point that is measured on an artifact, a constant distance is maintained between the sensor and the artifact. Unlike the traditional scanning method, using the constant standoff scanning method causes no inherent error due to misalignment or measurement angle. That is, using a perfect sensor and a perfect artifact, the measurement results will also be perfect. In practice, constant distance scanning was achieved by using a scan path defined by a reference model and also by an in-situ feedback control system based on real-time scan data. Depending on the application, the means of achieving constant distance scanning may be more appropriate than others.

While the constant standoff measurement method offers a significant improvement over a non-constant measurement methodology, this new method also has limitations. Small measurement errors do exist once we examine the more realistic scenario in which the part contains size and form defects. In this instance, we must determine the magnitude of the measurement errors as they relate to misalignment, measurement angle, and the size of the part defects. Let us again examine a base case in which we assume we have a measurement angle $\beta$ and some error in the measurement angle, $\beta_{\text {error }}$ (see Fig. 10). We also have some defect in the artifact geometry, represented by the "Error" surface in Figure 10. The distance between the part surface and the "Error" surface is represented as $x$ in equation (5). In practical applications, this "Error" surface would manifest itself as bumps, dents, pitting, or other defects in the manufacturing of the measurement part.

$$
\begin{aligned}
l & =\frac{\text { standoff } \times \cos \left(\beta_{\text {error }}\right)-x}{\cos \left(\beta_{\text {error }}\right)} \\
l^{\prime} & =\text { standoff }-l \\
\Delta x & =l^{\prime} \times \sin (90-\beta) .
\end{aligned}
$$

Following Figure 10 and equations (5)-(7) it can be seen that there will be error in a measurement such that the measured point will be located at a distance $(\Delta x, \Delta y)$ from the true measurement location. The magnitude of $\Delta x$ and $\Delta y$ will change with the factors of misalignment, measurement angle and the size of part defects. For

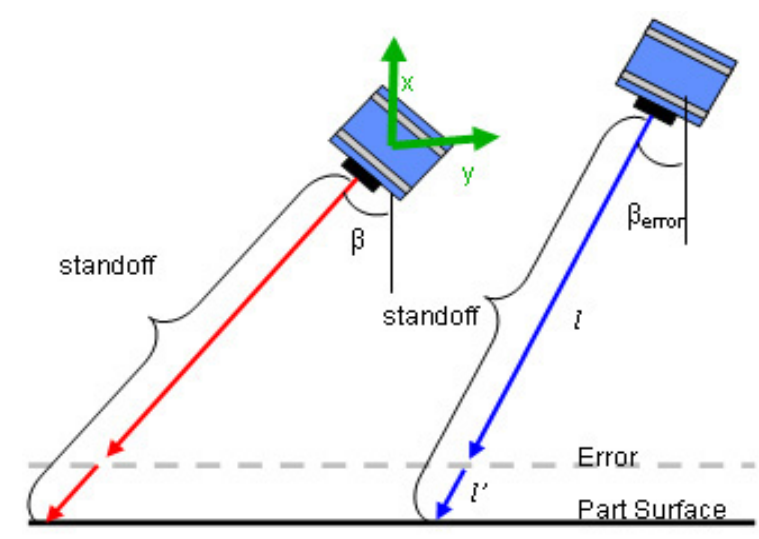

Fig. 10. Measurement errors caused by misalignment and artifact errors using constant standoff scanning.

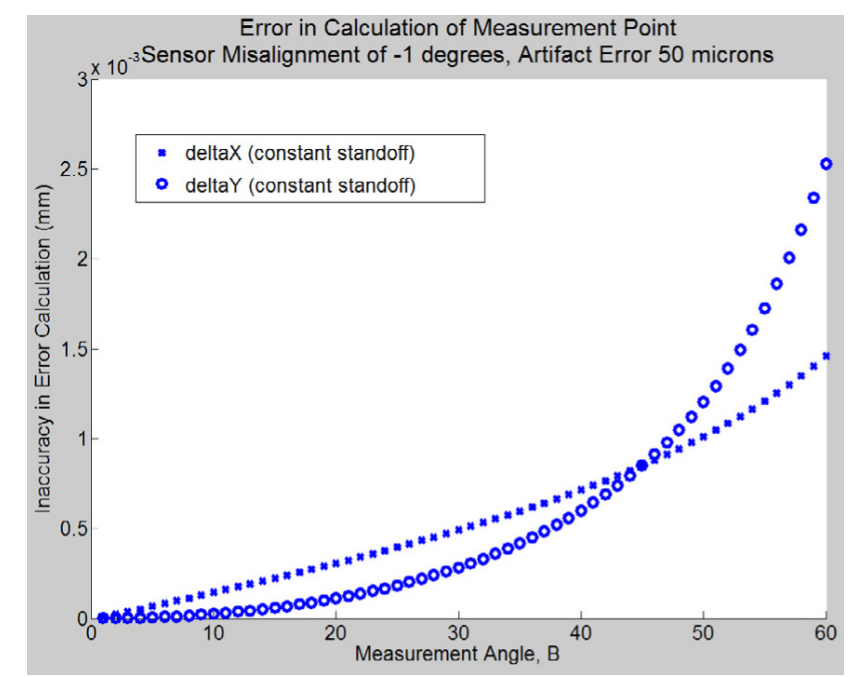

Fig. 11. (Constant Standoff): Effect of measurement angle on the measurement error, $\Delta x$ and $\Delta y$, with a misalignment of $-1^{\circ}$.

analysis, we have chosen the error surface to be a distance of $0.05 \mathrm{~mm}$ from the ideal artifact surface. For micron scale dimensional measurements, it is believed that reasonable artifact errors will not exceed $0.05 \mathrm{~mm}$. This distance will provide a conservative estimate of measurement error.

The influence of measurement angle on $\Delta x$ and $\Delta y$ can be seen in Figure 11 above. The measurement errors, $\Delta x$ and $\Delta y$, are seen to increase as a function of measurement angle. Similarly, $\Delta x$ and $\Delta y$, are seen to increase as a function of misalignment. Furthermore, it can be deduced from equations $(5)-(7)$ that as the size of the part defects decrease, the size of $\Delta x$ and $\Delta y$ will also decrease in a linear fashion and approach zero error.

Analysis of the measurement errors associated with constant distance scanning methods show that this method provides significantly more accurate measurement results than the traditional method using a non-constant standoff. Using the constant distance scanning method, the factors of misalignment, measurement angle, and artifact defects may still contribute some measurement 
errors. Examining a pragmatic "worst case" scenario in which misalignment is $1^{\circ}$, measurement angle is $45^{\circ}$, and part defects are $0.05 \mathrm{~mm}$, the measurement errors, $\Delta x$ and $\Delta y$, will be on the scale of $0.85 \mu \mathrm{m}$.

\section{Conclusions}

Current, traditional scanning methodologies are insufficient for achieving accurate micron-scale measurements using many non-contact dimensional measurement systems. The traditional scanning methodologies, coupled with point sensor misalignment, large measurement angles and registration software have the potential to introduce significant measurement error. The presence of this substantial error has been demonstrated through both experimentation and simulation.

Some degree of sensor misalignment is inherent to any measurement system. Accurate determination of the point sensor beam alignment with respect to the measurement system coordinate axes may prove difficult if not impossible. Additionally, in order to measure parts with complex geometry it is necessary to use a non-contact measurement system at large measurement angles.

Thus, in order to achieve sub-micron measurement errors it is necessary to scan an artifact while maintaining a constant distance between the sensor and the measurement part. This method allows the measurement system to operate in a manner similar to a CMM. Analysis of the constant standoff measurement method shows that the factors of misalignment, measurement angle, and artifact defects contribute a combined measurement error of one micron or less in typical applications.

Work is currently being performed to develop and implement constant distance scanning methods. Experimental data from constant distance scanning can be compared with the theorized results to validate constant distance scanning as a solution to the measurement errors explored this paper. Furthermore, future work is planned to determine the effect of additional error sources, such as sensor noise, on the overall measurement accuracy.

Acknowledgements. This work was supported by the National Science Foundation under Award Number EEC-9529125.

\section{References}

1. A. Weckenmann, X. Jiang, K.D. Sommer, U. NeushaaeferRube, J. Seewing, L. Shaw, T. Estler, Multisensor data fusion in dimensional metrology, CIRP Annals 58, 701-721 (2009)
2. F. Blais, A Review of 20 years of range sensor development, J. Electron. Imag. 13, 231-240 (2004)

3. K. Harding, Latest optical methods for industrial dimensional metrology, Proc. SPIE 6000, 1-14 (2005)

4. K.-C. Fan, A non-contact automatic measurement for freeform surface profiles, Comput. Integr. Manufact. Syst. 10, 277-285 (1997)

5. J.C. Ziegert, C.D. Mize, The laser ball bar: a new instrument for machine tool metrology, Precision Eng. 16, 259-267 (1994)

6. L. Zhu, J. Barhak, V. Sirvatsan, R. Katz, Error analysis and simulation of a four-axis optical inspection system, in Proc. of the DET 2004 Conference, Washington DC, 2004

7. V. Srivatsan, R. Katz, D. Dutta, Fixtureless sensor standoff control for high-precision dimensional inspection of freeform parts, J. Manufact. Sci. Eng. 129, 172-179 (2007)

8. S. Son, H. Park, K. Lee, Automated laser scanning system for reverse engineering and inspection, Int. J. Machine Tools Manufact. 42, 889-897 (2007)

9. Prieto, T. Redarce, P. Boulanger, R. Lepage, CAD-based range sensor placement for optimum 3D data acquisition, in Proc. of the Second International Conference on 3-D Digital Imaging and Modeling, Ottawa, Ont., 1999, pp. $128-137$

10. C. Mehdi-Souzani, F. Thiebaut, C. Lartique, Scan planning strategy for a general digitized surface, J. Comput. Inform. Sci. Eng. 6, 331-339 (2006)

11. H-Y. Feng, Y. Liu, F. Xi, Analysis of digitizing errors of a laser scanning system, J. Int. Soc. Precision Eng. Nanotechnol. 25, 185-191 (2001)

12. A. Isheil, J.-P. Gonnet, D. Joannic, J.-F. Fontaine, Systematic error correction of a 3D laser scanning measurement device, Opt. Lasers Eng. 49, 16-24 (2011)

13. S. Martinez, E. Cuesta, J. Barreiro, B. Alvarez, Methodology for comparison of laser digitizing versus contact systems in dimensional control, Opt. Lasers Eng. 48, 1238-1246 (2010)

14. J. Barhak, R. Katz, Interpretation of laser measurements produced by the reconfigurable inspection machine using the "Virtual ball" method, in Proc. of CIRP 2nd Intl. Conference on Reconfigurable Manufacturing Systems, Ann Arbor, MI, 2003

15. Z. Warlick, Development of technologies required for a prototype high-accuracy, high-density, non-contact dimensional inspection system, Master thesis, University of Michigan, 2007

16. Zhu, J. Barhak, V. Srivatsan, R. Katz, Efficient registration for precision inspection of free-form surfaces, Int. J. Adv. Manufact. Technol. Marchine 32, 505-515 (2007)

17. D. Steiner, Calibration With Respect to the Beam Direction of a Non Contact Point Sensor, in Proc. of the ASME International Mechanical Engineering Congress and Exposition, Chicago, Illinois, 2006 